\section{Intrathecal Baclofen Administration During Pregnancy: A Case Series and Focused Clinical Review}

Christina M. Morton, MD, Joshua Rosenow, MD, Cynthia Wong, MD, Kristi L. Kirschner, MD

\section{INTRODUCTION}

Literature regarding intrathecal baclofen (ITB) therapy during pregnancy is sparse, especially that documenting outcomes of pregnancies in women exposed to ITB from the time of conception [1-4]. Because of the lack of controlled, prospective studies of ITB administration in pregnancy, most practicing clinicians rely on previous experience in management of these unusual cases. The objective of this retrospective study and focused clinical review is to address detailed management of ITB therapy and complications related to ITB administration during pregnancy such as preconceptual counseling, the effect of ITB on the neonate, pump placement and replacement, Caesarean deliveries, and anesthesia management. The management and outcomes of 4 pregnancies with continuous ITB therapy from the time of conception are presented, and previously reported cases are reviewed.

\section{CASE PRESENTATIONS}

Four pregnancies in 3 women with spasticity treatment by ITB were identified from an institutional review board-approved chart review of 755 charts of women seen in a multidisciplinary specialty clinic for women with disabilities. Maternal clinic, allied health, labor/delivery, and anesthesia records were reviewed from the time of pregnancy diagnosis to 6 weeks postpartum. The corresponding neonatal inpatient medical records were also reviewed.

\section{Case 1}

A 27-year-old G5P3022 ( $G=$ number of pregnancies; P [T/P/A/L $]=$ number of pregnancies a woman has carried to viability, $\mathrm{T}=$ term deliveries, $\mathrm{P}=$ preterm deliveries, $\mathrm{A}=$ abortions [includes elective, spontaneous, ectopics, molar pregnancies-anything less than 20 weeks], $\mathrm{L}=$ living) black woman with $\mathrm{T} 5$ incomplete spinal cord injury (SCI) secondary to a gunshot wound at age 19, history of autonomic dysreflexia (AD), and spontaneous voids with intact perineal sensation, had an ITB pump placed 6.5 years before pregnancy diagnosis for intractable spasticity poorly controlled by oral antispasmodics. The pump was placed in a subcutaneous pocket of the left anterior abdomen with the tunneled intrathecal catheter tip located at T10. Her ITB dose was $200 \mu \mathrm{g} /$ day at the time her pregnancy was confirmed at 12 weeks' gestation, and a scheduled pump revision was delayed. At 37 weeks she had spontaneous onset of labor with a spontaneous vaginal delivery. Epidural analgesia was initiated at the L5-S1 interspace with bupivacaine and fentanyl maintained with a continuous infusion throughout labor. Her blood pressure ranged from 120/70 to 150/85 $\mathrm{mm} \mathrm{Hg}$ and her heart rate from 90 to $100 \mathrm{bpm}$. There were no other symptoms of AD (Table 1). She underwent a postpartum tubal ligation under epidural anesthesia using the in situ epidural catheter. An ITB pump revision was scheduled, and she remained on her initial ITB dose of $200 \mu \mathrm{g} / \mathrm{day}$.
C.M.M. Departments of Physical Medicine and Rehabilitation and Obstetrics and Gynecology, University of Michigan Medical School, $325 \mathrm{E}$. Eisenhower Pkwy, Suite 100, Ann Arbor, Ml 48108. Address correspondence to: C.M.M.; e-mail: cmmorton@med.umich.edu Disclosure: nothing to disclose

J.R. Functional Neurosurgery, Department of Neurosurgery, Northwestern University Feinberg School of Medicine, Chicago, IL Disclosure: 2A, Boston Scientific Neuromodulation and Medtronic Navigation

C.W. Division of Obstetrical Anesthesia, Department of Anesthesiology, Northwestern University Feinberg School of Medicine, Chicago, IL Disclosure: nothing to disclose

K.L.K. Department of Physical Medicine and Rehabilitation, RIC Women with Disabilities Center, Northwestern University Feinberg School of Medicine, Chicago, IL

Disclosure: 3A, honoraria for academic talks; $5 A$; 9, editor of PM\&R Ethical Legal Feature

Disclosure Key can be found on the Table of Contents and at www.pmrjournal.org

A poster was presented at the AAPM\&R Assembly in September of 2007. No funding was designated for this study.

Submitted for publication April 8, 2009; accepted July 22. 
Table 1. Perinatal outcomes

\begin{tabular}{|c|c|c|c|c|c|c|c|c|c|}
\hline $\begin{array}{l}\text { Case } \\
\text { No. }\end{array}$ & $\begin{array}{c}\text { Gestational } \\
\text { Age (wk) }\end{array}$ & $\begin{array}{c}\text { Birth-weight } \\
\text { (g) }\end{array}$ & $\begin{array}{c}\text { Apgar } \\
\text { Scores* }\end{array}$ & Delivery & Anesthesia & Size $^{\dagger}$ & Infant Complications & LOS & BF \\
\hline 1 & 37 & 3799 & 8,9 & SVD & Epidural & LGA & & 2 & $\overline{\text { Yes }}$ \\
\hline $2 a$ & $394 / 7$ & 2560 & 9,9 & SVD & Systemic opioid & SGA & $\begin{array}{l}\text { Monitoring for suspected } \\
\text { sepsis due to GBS+, } \\
\text { jaundice }\end{array}$ & 2 & No \\
\hline $2 b$ & $355 / 7$ & 2210 & $2,6,8$ & SVD & Systemic opioid & $A G A$ & $\begin{array}{l}\text { Tight nuchal and body } \\
\text { cord } \times 1, \mathrm{O}_{2} \text { per mask, } \\
\text { naloxone IV, SCN for } \\
\text { prematurity, jaundice }\end{array}$ & 2 & No \\
\hline 3 & $341 / 7$ & 2240 & 9,9 & CS & General & $A G A$ & $\begin{array}{l}\text { CS indicated for } \\
\text { maternal preeclampsia, } \\
\mathrm{O}_{2} \text { with NCPAP, SCN for } \\
\text { prematurity }\end{array}$ & 9 & No \\
\hline
\end{tabular}

SVD = spontaneous vaginal delivery; $C S=$ Caesarean section; $A G A=$ appropriate for gestational age; $L G A=$ large for gestational age; SGA = small for gestational age; GBS + = positive for Group B Streptococcus; SCN = special care nursery; NCPAP = nasal continuous positive airway pressure ventilation; LOS $=$ length of stay (infant hospitalization); $\mathrm{BF}=$ breastfed.

*Apgars reported at 1, 5 and 10 minutes after birth.

${ }^{\dagger}$ Size reported per BMC Pediatrics Fetal-Infant Growth Chart for Preterm Infants.

\section{Case 2a}

A 21-year-old G2P1010 black woman with spastic athetoid dystonic cerebral palsy (CP) resulting in tetraparesis, right greater than left dystonic posturing, spastic bladder, and dysarthria had an ITB pump placed subcutaneously in the right lower abdomen with the catheter tip at T8 level 4 years before her pregnancy diagnosis. Her ITB dose was $200 \mu \mathrm{g} /$ day at pregnancy diagnosis at 8 weeks' gestation. She remained on the same ITB dose during her pregnancy. She had spontaneous onset of labor at 39 4/7 weeks' gestation. Labor analgesia was provided with systemic opioids, as the anesthesia providers were uncomfortable breaching her neuraxial space (Table 1). Two weeks postpartum, she reported increased spasticity with discomfort. An abdominal radiograph showed no catheter abnormalities. Her ITB dose was increased to $220 \mu \mathrm{g} /$ day and she remained on this dose for 6 weeks postpartum.

\section{Case 2b}

Through the next 2.5 years the same patient as case $2 \mathrm{a}$ had intermittent titrations of her ITB to a dose of $400 \mu \mathrm{g} / \mathrm{day}$, requiring pump reservoir refills every 2 months. After confirmation of her second pregnancy at $6+$ weeks' gestation, she experienced increased spasticity and her ITB dose was raised from $400 \mu \mathrm{g}$ to $430 \mu \mathrm{g} /$ day. She remained on this dose with spasticity well controlled through 2 routine refills. At 35 $5 / 7$ weeks' gestation, she had preterm labor with preterm rupture of membranes (Table 1). The infant initially had fair respiratory effort, but subsequently experienced tachypnea and hypoxemia. Oxygen was administered by face mask for 12 minutes, after which she was successfully weaned to room air. As a result of increased spasticity at 1 week postpartum, the patient's ITB dose was adjusted to $465 \mu \mathrm{g} /$ day, and she remained on this dose after undergoing a tubal ligation at 6 weeks postpartum.

\section{Case 3}

A 35-year-old G2P1010 Hispanic woman with spastic athetoid CP resulting in spastic tetraparesis, neurogenic bladder/ bowel with chronic constipation, bilateral hip and shoulder reconstructions with remote history of right hip osteomyelitis, severe kyphoscoliosis with laminectomy/fusion ( $\mathrm{Tl}$ through the sacrum) and Harrington rods placed at age 15, had an ITB pump placed in a subcutaneous pocket of her right lower abdomen (undocumented catheter tip level because of spinal hardware artifact) 2 years before her pregnancy diagnosis. Pregnancy was confirmed at 5 weeks' gestation while receiving an ITB dose of $785 \mu \mathrm{g} /$ day. At 8 weeks' gestation, after weaning from oral diazepam and dantrolene, her ITB dose was increased to $795 \mu \mathrm{g} /$ day. She remained on .5 to 1 tablet hydrocodone/acetaminophen every evening for management of hip and back pain. At 28 weeks' gestation, her pump was noted to have shifted posterolaterally as a result of her gravid abdomen, but remained in the subcutaneous right lower quadrant. Because of severe contractures and spasticity the patient was deemed to be a poor candidate for a vaginal delivery, and elective Caesarean delivery was planned. During an antepartum anesthesiology evaluation, general anesthesia was planned because of her previous spinal fusion. At 33 weeks' gestation the patient experienced increasing tachypnea and hypoxemia (documented on overnight pulse oximetry) believed to be related to her kyphoscoliosis as well as spasticity of her diaphragm, chest wall, and accessory muscles. She was found to have hypertension and was admitted directly to the labor and delivery unit. She was diagnosed with severe preeclampsia and treated with intravenous magnesium sulfate prophylaxis. She underwent urgent Caesarean delivery at 34 1/7 weeks' gestation after awake fiberoptic-assisted intubation and induction of general anesthesia (Table 1). The infant displayed accessory respiratory muscle retractions shortly after birth and was treated with positive-pressure ventilation by nasal continuous posi- 
tive airway pressure (CPAP) with subsequent weaning to room air within 24 hours. Difficulties with Caesarean delivery wound closure necessitated intraoperative general surgery service consultation. The patient was extubated in the operating room at the end of the procedure; however, after surgery the mother displayed signs of respiratory distress, required oxygen supplementation, and was transferred to the medical intensive care unit (MICU) for observation. Her ITB dose was lowered and maintained at $780 \mu \mathrm{g} /$ day while in the MICU to prevent further possible ITB-associated respiratory depression. The mother was able to successfully wean from oxygen within a few days after her hospital discharge and was restarted on her pre-pregnancy medications and thus declined breastfeeding.

\section{DISCUSSION}

Four separate case reports documenting 5 pregnancies have been previously reported, describing general pregnancy and infant outcomes after ITB therapy during pregnancy [1-4]. Two of the cases reported a short course of ITB treatment during the third trimester, one documenting an ITB programmable pump implanted at 30 weeks' gestation and another, ITB administration for management of severe tetanus at 28 weeks' gestation for 3 weeks' duration. All pregnancies resulted in preterm births (32-36 weeks) of low birth weight infants (1500-2155 g) appropriate for (preterm) gestational age and 5-minute Apgar scores of 10. No infants were reported to have evidence of teratogenicity, neurologic, or respiratory complications at birth, and two male infants born to one woman were documented to have normal psychomotor development to the ages of 12 and 24 months. There were no reported maternal adverse side effects or pump mechanical malfunctions.

The addition of this study to the existing literature increases by threefold the number of cases reporting fetal ITB exposure from the time of conception. Within a hospitalbased clinic population, 3 women were identified with 4 subsequent births who had ITB administration by implanted programmable pumps starting at least 2 years before conception. In this series, only 2 of the 4 infants were born preterm, one by urgent Caesarean delivery for maternal preeclampsia and the other a spontaneous vaginal delivery. Both preterm infants were of appropriate size and weight for (preterm) gestational age; the full-term infants were small and large for gestational age. Both preterm infants were of low birth weight (less than $2500 \mathrm{~g}$ ), treated for tachypnea with oxygen supplementation by mask or nasal CPAP, and transferred to the special care nursery for prematurity (Table 1).

One mother breastfed her infant. Therapeutic oral doses of baclofen are known to pass into breast milk, although it is not known whether detectable levels pass into breast milk in mothers receiving ITB. American Academy of Pediatrics (AAP) guidelines classify baclofen as "usually compatible with breastfeeding" [5]. No adverse infant reactions were documented for this infant during the course of breastfeeding.

\section{ITB Pharmacokinetics and Administration}

ITB is indicated for the treatment of spasticity of central nervous system origin in individuals with spinal cord injury, dystonia, spastic diplegia, cerebral palsy, multiple sclerosis, amyotrophic lateral sclerosis, traumatic brain injury, and trigeminal neuralgia [6]. The dose of ITB necessary to slow down the reflex circuit is variable but is generally at least 100 to 1000 times smaller than the oral dose, acting directly and selectively at the spinal level [7]. The therapeutic dose required to treat spasticity of cerebral origin is about 3 times higher than that used to treat spasticity of spinal origin [8]. After lumbar administration, baclofen migrates upward, but the concentration declines along the neuraxis so that the lumbar/cistern ratio is approximately 4:1 [9]. The systemic absorption is less than $1 \%$ of the intrathecal dose [10]. In ITB therapy, effective spasticity treatment can be maintained with significantly lower blood serum levels compared with therapeutic oral baclofen therapy, with suspected lower overall fetal exposure and thus decreased risk to the fetus for teratogenicity or adverse neonatal outcomes.

Abrupt discontinuation of either oral or intrathecal baclofen is associated with withdrawal symptoms, most commonly including hallucinations and seizures. Other withdrawal symptoms include itching, high fever, altered mental status, exaggerated rebound spasticity, and muscle rigidity that in rare cases can progress to rhabdomyolysis, multiple organ-system failure, and death. Signs of overdose may be sudden or insidious, with acute massive overdose presenting as a coma, and less severe forms of overdose presenting as drowsiness, lightheadedness, dizziness, somnolence, respiratory depression, seizures, rostral progression of hypotonia, or loss of consciousness progressing to coma [6].

\section{Effects of ITB on the Neonate}

Baclofen crosses the placenta, and its use during pregnancy must be weighed against possible benefit to the mother versus potential risk to the fetus. Baclofen is listed in U.S. Food and Drug Administration pregnancy category $\mathrm{C}$ and has not been adequately studied during human pregnancy. When given orally to rats, baclofen has been shown to increase the incidence of fetal omphaloceles (ventral hernias) when administered in excess of the maximum recommended oral dose for humans $[6,11]$. Pregnancy is associated with physiologic changes to neuraxial anatomy (eg, decrease in cerebrospinal fluid volume [CSF] ) and changes in CSF composition (eg, pH and protein concentration) [12]. It is unknown how these changes affect the bioavailability of ITB during pregnancy. No known adverse neonatal outcomes have been reported in association with ITB therapy during pregnancy $[13,14]$. Infant withdrawal symptoms have not been previously reported in ITB administration during pregnancy and is not reported in this case series. However, there has been one report of perinatal generalized convulsions 7 days after delivery after high-dose maternal oral baclofen administration (20 mg 4 times daily) throughout pregnancy. The neonate's seizures did not respond to typical treatment 
with benzodiazepines and antiepileptics, but did stop within 30 minutes after an intravenous baclofen dose [14].

\section{ITB Management and Considerations in Pregnancy}

Preconceptual counseling before programmable baclofen pump placement and in women with ITB pumps already implanted is fundamental to ensuring proper preparation and management throughout pregnancy and the peripartum period. In case number 3 , the physiatrist educated the patient about possible risks and benefits regarding effects to both her and her future offspring of ITB therapy, including risk and benefits of alternative methods for spasticity management. Side effects should be discussed; lethargy, fatigue, and hypotension may be potentially more difficult to manage during pregnancy. This population may also be at higher risk for respiratory depression with administration of these medications during pregnancy owing to kyphoscoliosis or concomitant neuromuscular weakness, and drugs administered for labor analgesia (eg, opioids) may potentiate side effects such as respiratory depression.

The patient should be educated about possible pump mechanical malfunction, and plans for careful monitoring should be developed. Further studies are needed to determine prevalence and risk for these complications in pregnant women. Previously reported complications in nonpregnant patients include device or component failure; change in flow performance; inability to program the device because of programmer failure; inaccessible refill port as a result of inverted pump or catheter access port failure; pocket seroma, hematoma, erosion, or infection; post-lumbar puncture headache (spinal headache); CSF leak; radiculitis; arachnoiditis; bleeding; spinal cord damage; meningitis (intrathecal applications); anesthesia complications; damage to the pump, catheter, and catheter access system because of improper handling and filling before, during, or after implantation; change in catheter performance caused by catheter kinking, disconnection, leakage, breakage, occlusion, dislodgement, migration, or catheter fibrosis; body rejection phenomena; surgical replacement of pump or catheter as a result of complications; possible development of an inflammatory mass at the tip of the catheter; changes in bowel or bladder management; and possible adverse effects of environmental temperature on pump function (such as in cases of short-wave diathermy or magnetic resonance imaging) $[9,15-17]$.

\section{Pump Placement and Replacement}

The upper border of the pump is usually positioned at approximately the level of the umbilicus. The change in abdominal shape during pregnancy can cause complications. As the uterus expands, the pump position will be altered. This may cause the patient some discomfort if the pump begins to impact the iliac crest, which is usually just lateral to the pump. Moreover, if the shape of the gravid abdomen causes the pump to tilt at an awkward angle, it is possible that the pump will be more prone to eroding through the overly- ing subcutaneous fat and come closer to the skin. This can not only cause discomfort but the pump may need to be operatively repositioned if the skin appears threatened and the pump is in danger of being exposed. Most implanting surgeons leave a significant amount of extra catheter coiled behind the pump. Although this guards against problems with catheter dislodgement as the abdominal girth (and thus the length of catheter required to traverse the flank from pump to spine) increases, it still leaves the catheter at risk for kinking as the abdomen changes shape. Given the serious consequences of abrupt ITB withdrawal, patients should be reminded of the warning signs and symptoms of this condition and instructed to seek attention with the treating center immediately should any of these appear.

As pumps do require replacement because of end of life of the implanted battery, patients considering becoming pregnant should discuss this with their ITB treatment team. The treatment team can assess how close the patient is to requiring replacement. If it appears the pump has less than a year left, it may be prudent to prophylactically replace the pump so that the patient and developing fetus do not have to undergo the procedure during the pregnancy.

\section{Caesarean Section Deliveries}

Obstetricians performing caesarean deliveries in patients with implanted pumps need to be aware of the location of the pump and surrounding pocket. Care should be taken during the approach to the uterus to avoid entering the pump pocket. Should any catheter be observed in the operative field, it should be protected during the procedure and inspected afterward for damage. Importantly, it should be left with only gentle bends and without tight coils or kinks, so that ITB administration is not hindered. The morphology of the pump system may be assessed via plain radiographs. Patients who have not had these at the time of pump implantation should have them before they conceive so that a record exists for use by the treating obstetric and anesthesia teams.

\section{Anesthesia Management}

Anesthesiologists providing obstetric anesthesia to patients with implanted intrathecal catheters should be aware of the course of the catheter, level of entry into the thecal sac, and level of the catheter tip. Although there is no absolute contraindication to spinal or epidural anesthesia with concomitant ITB therapy, care should be taken not to pierce the catheter with a needle used for administering these therapies [18]. Epidural and dural puncture should be performed remote from the catheter insertion site. Theoretically it may be possible to administer spinal anesthesia via the intrathecal catheter; however, use of this technique has not been reported. The catheter may be accessed via the small catheter access port. After withdrawing several milliliters of CSF to ensure that the catheter is emptied of drug, the spinal anesthetic may be infused, followed by a sterile saline flush. The pump may then be reprogrammed to refill the catheter and 
continue ITB therapy. This technique might have allowed spinal anesthesia for patient 3 (which was not otherwise possible because of spinal fusion). However, this technique has not been reported.

\section{CONCLUSIONS}

It is not possible to draw conclusions about pregnancy outcomes in patients with ITB therapy from these few cases. No factors currently dictate against pregnancy for patients with ITB pumps already in place. Further studies, preferably longterm prospective ones, are recommended to provide more data to investigate maternal and infant risk of complications, and to improve guidance for clinical decisions in terms of care for these individuals.

\section{ACKNOWLEDGMENTS}

The authors thank Joan T. Le, MD, for her assistance with data collection and manuscript editing.

\section{REFERENCES}

1. Delhaas EM, Verhagen J. Pregnancy in a quadriplegic patient treated with continuous intrathecal baclofen infusion to manage her severe spasticity. Case report. Paraplegia 1992;30:527-528.

2. Muñoz FC, Marco DG, Pérez AV, Camacho MM. Pregnancy outcome in a woman exposed to continuous intrathecal baclofen infusion. Ann Pharmacother 2000;34:956.

3. Engrand N, Van de Perre P, Vilian G, Benhamou D. Intrathecal baclofen for severe tetanus in a pregnant woman. Eur J Anaesthesiol 2001;18: 261-263.

4. Roberts AG, Graves CR, Konrad PE, et al. Intrathecal baclofen pump implantation during pregnancy. Neurology 2003;61:1156-1157.

5. Ward RM, Bates BA, Benitz WE, et al. The transfer of drugs and other chemical into human milk. Pediatrics 2001;108:776-789.
6. Baclofen injection. Drug insert. Norvartis Pharma AG Basle, Switzerland for Medtronic, Inc. Minneapolis, MN, 2002.

7. Albright AL, Schultz BL. Plasma baclofen levels in children receiving continuous intrathecal baclofen infusion. J Child Neurol 1999;91:733736.

8. Koulousakis A, Kuchta J. Intrathecal antispastic drug application with implantable pumps: results of a 10 year follow-up study. Acta Neurochir Suppl 2007;97:181-184.

9. Gracies JM, Nance P, Elovic E, McGuire J, Simpson DM. Traditional pharmacologic treatments for spasticity. Part II: General and regional treatments. Muscle Nerve Suppl 1997;6:S92-S120.

10. Kroin JS, Ali A, York M, Penn RD. The distribution of medication along the spinal canal after chronic intrathecal administration. Neurosurgery 1993;33:226-230

11. Meadows M. Pregnancy and the drug dilemma. FDA Consumer magazine. Volume 35, Number 3; May-June 2001. Available at: http:// www.fda.gov/fdac/features/2001/301_preg.html\#categories. Accessed January 27, 2009.

12. Chang $A B$. Physiologic changes of pregnancy. In: Chestnut DH, ed. Obstetric Anesthesia Principles and Practice. Philadelphia, PA: Elsevier; 2004, 15-36

13. Moran LR, Almeida PG, Worden $S$, Huttner KM. Intrauterine baclofen exposure: a multidisciplinary approach. Pediatrics 2004;114:e267e269.

14. Ratnayaka BDM, Dhaliwal H, Watkin $S$. Drug points: neonatal convulsions after withdrawal of baclofen. BMJ 2001;323:85.

15. Plassat R, Verbe BP, Menei $P$, Menegalli D, Mathe JF, Richard I. Treatment of spasticity with intrathecal baclofen administration: longterm follow-up, review of 40 patients. Spinal Cord 2004:42:686-693.

16. Guillaume D, Van Havenburg A, Vloeberghs M, Vidal J, Roeste G. A clinical study of intrathecal baclofen using a programmable pump for intractable spasticity. Arch Phys Med Rehabil 2005;86:1265-1271.

17. Synchromed II drug infusion system brief summary: ITB therapy for severe spasticity. January 2008. Available at: http://www.medtronic. $\mathrm{com} /$ physician/itb/disclosure-brief-summary2.html. Medtronic Inc, 2008. Accessed January 28, 2009.

18. Tarshis J, Zuckerman JE, Katz NP, Segal S, Mushlin PS. Labour pain management in a parturient with an implanted intrathecal pump. Can J Anaesth 1997;44:1278-1281. 